\title{
Efectos Genéticos Directo y M aterno sobre el Crecimiento de Ovinos de la Raza Junín
}

\author{
Direct and Maternal Genetic Effects on the Growth of Junín \\ SHEEP BREED
}

Diógenes Valerio ${ }^{1,2,3}$, Gustavo Gutiérrez ${ }^{2,4}$, Juan Chávez ${ }^{2,5}$

\section{Resumen}

\begin{abstract}
Con el objetivo de estimar los efectos genéticos - directo y materno - vinculados a la expresión de características de crecimiento en corderos de la raza Junín, se aplicaron cuatro modelos mixtos en el estudio de sus pesos de nacimiento (PN), destete (PD), primera esquila (PE) y ganancia diaria del nacimiento al destete (GPND), a través de análisis univariado y bivariado. Los modelos usados en análisis univariado incluyeron como efectos fijos el año de nacimiento y sexo, y como covariables el peso de la madre al empadre (para PN y PD), la edad del cordero al destete (para PD y GPND) y la edad a la primera esquila (para PE). El Modelo 1 incluyó los efectos fijos y el efecto genético aditivo directo (a); el Modelo 2, similar al 1, adicionó el efecto genético aditivo materno (m), considerando la $\mathrm{Cov}_{\mathrm{am}}=0$; en el Modelo 3, igual al 2, se asumió la $\mathrm{Cov}_{\mathrm{am}}=\mathrm{A} \sigma_{\mathrm{am}}$; y el Modelo 4, igual al 3, se adicionó el efecto del ambiente permanente materno (c). Los modelos para el análisis bivariado fueron extensiones del univariado, empleándose en todos los análisis el método de máxima verosimilitud restringida (REML), instrumentado en el programa ASReml. En el análisis univariado, el Modelo 2, y en el bivariado el Modelo 3, estimaron mejor los parámetros genéticos, incluidas las correlaciones entre los efectos aditivos directos y maternos para las características. Los valores estimados de heredabilidad variaron entre bajos y moderados, indicando la posibilidad de ser aplicados en planes de mejora genética del ovino Junín, los mismos que incrementarían su eficiencia al adicionarse a ellos los valores, entre moderados y altos, de las correlaciones genéticas y fenotípicas estimadas.
\end{abstract}

Palabras clave: crecimiento; parámetros genéticos; efecto materno; raza Junín

${ }^{1}$ Facultad de Ciencias Agropecuarias, Universidad Nacional Daniel Alcides Carrión, Pasco, Perú. Artículo científico derivado de la tesis del Sr. Diógenes Valerio, para optar el grado de trabajo de Magister Scientiae en Producción Animal de la Universidad Nacional Agraria La Molina, Lima, Perú

${ }^{2}$ Facultad de Zootecnia, Departamento de Producción Animal, Universidad Nacional Agraria La Molina, Lima, Perú

${ }^{3}$ E-mail:dlvlal@hotmail.com

${ }^{4}$ Responsable de la correspondencia.E-mail: gustavogr@lamolina.edu.pe

${ }^{5}$ E-mail: jchavez@lamolina.edu.pe

Recibido: 11 de junio de 2014

Aceptado para publicación: 4 de noviembre de 2014 
In order to estimate the genetic effects - direct and maternal - linked to the expression of growth traits in lambs of Junín breed, four mixed models were applied in the study of the birth weight (PN), weaning weight (PD), first shearing weight (PE) and daily body weight gain from birth to weaning (GPND), through univariate and bivariate analysis. The models used in univariate analysis included as fixed effects year of birth and sex, and as covariates the mother's weight at mating (for PN and PD), age of lamb at weaning (for PD and GPND), and age at first shearing (for PE). Model 1 included the fixed effects and the direct additive genetic effect (a); Model 2, similar to 1, added additive maternal genetic effect $(m)$, considering $\operatorname{Cov}_{\mathrm{am}}=0$; Model 3, similar to 2, assumed $\operatorname{Cov}_{\mathrm{am}}=\mathrm{A} \sigma_{\mathrm{am}}$; and Model 4, equal to 3, added the maternal permanent environmental effect $(c)$. All bivariate models were extensions of the univariate ones. In all the analyses, the restricted maximum likelihood (REML) method, implemented in the ASReml program was applied. Univariate Model 2 and bivariate Model 3 estimated the best genetic parameters, including correlations between direct and maternal additive effects for the traits. The estimated heritability values ranged from low to moderate, indicating the possibility of its application in genetic improvement plans for Junín sheep, which would increase its efficiency when the estimated, moderate to high genetic and phenotypic correlations are also included.

Key words: growth; genetic parameters; maternal effect; Junín breed

\section{INTRODUCCIÓN}

Uno de los factores para el éxito económico de la crianza de ovinos a nivel comercial es el potencial de crecimiento de los corderos, y esto depende del genotipo del animal, de su madre y del ambiente en que se desarrollan (Mueller, 2001; Momoh et al., 2013). Los modelos de evaluación genética de corderos para la selección de reproductores, que no incluyen el efecto maternal, ofrecen estimados sesgados de sus valores genéticos o de cría, dado que las heredabilidades de las características empleadas en este proceso no son precisas (Ghafouri-Kesbi y Baneh, 2012). Por otro lado, los análisis univariados, bivariados o multivariados, empleados para obtener estimados más precisos de los parámetros genéticos, ofrecen diversos valores de heredabilidad (Bahreini Behzadi, 2013), lo cual debe considerarse en la selección multicarácter.
En la década de los 50, la Empresa Cerro de Pasco Corporation, entonces propietaria de los predios entregados a la SAIS Túpac Amaru, a través del proceso de reforma agraria de los años 70 (Ley $\mathrm{N}^{\circ}$ 17716), inició trabajos de selección para la formación de una raza ovina adaptada a la condiciones de crianza en pastoreo sobre praderas nativas de la sierra central del país. Para este fin, se emplearon como base borregas criollas y mestizas que fueron apareadas con reproductores de las razas Corriedale (Chile, Argentina, Estados Unidos) y Romney Marsh, Columbia, Panamá y Warhill. Esto fue seguido de un proceso de selección y apareamientos inter se en rebaño cerrado que dio lugar a la raza Junín, declarada como tal en el año 1973, por recomendación de la Dra. Helen Turner, investigadora en mejoramiento genético de ovinos del Commonwealth Scientific and Industrial Research Organization (CSIRO) de Australia (Linares et al., 2011). 
En la década de los 70, la población de esta raza permaneció casi estática, con cerca de 175000 animales a través de los años, a consecuencia de diversos factores (precios de la fibra y de la carne, capacidad de carga de los pastos, cambios en la dirigencia de la SAIS, injerencia del gobierno y problemas internos de relacionamiento cooperativo y comunitario), que afectaron también sus niveles de productividad (Valdivia y Pichihua, 1986). El periodo subsiguiente, desde mediados de la década de los 80 hasta principios de los 90, fue más inestable por los atentados desatados por grupos terroristas que, además, obligaron a la SAIS Túpac Amaru a distraer esfuerzos y presupuesto en gastos de seguridad (Sánchez, 1989).

Esto se ha venido revirtiendo progresivamente en los años posteriores, prestándose - entre otros - una mayor atención a los sistemas de manejo y a la mejora genética de la población. En este marco, el año 2010, con una población de 103 mil ovinos, se inició el registro genealógico oficial de los animales, a través de un programa que consta de una primera etapa (2010-2015), donde se establece un registro de fundación para los que cumplen con el estándar de la raza, y una segunda etapa - después de 2015 - para la formación del Libro de Pedigrí de la Raza Junín (Linares et al., 2011). Esta nueva realidad crea las condiciones para iniciar planes de mejoramiento genético basados en una adecuada caracterización de la población y en el desarrollo de instrumentos factibles de ser aplicados para una selección efectiva y eficiente.

Por estos motivos, el objetivo del presente trabajo fue estimar los efectos genéticos, tanto directos como maternales, relacionados con la expresión de características de crecimiento en corderos de la raza Junín, con la finalidad de aplicarlos en el diseño de programas de mejora genética.

\section{Materiales y Métodos}

Se trabajó con datos de la población ovina de la SAIS Túpac Amaru Limitada $\mathrm{N}^{\circ}$ 1 , empresa ganadera dedicada a la crianza de ovinos Junín, en la sierra central del Perú (Junín), ubicada a una altitud de 3800 msnm.

Entre 1982 y 1984, investigadores del Programa de Mejoramiento Animal de la Universidad Nacional Agraria La Molina, en el marco del Programa Colaborativo de Apoyo a la Investigación en Rumiantes Menores (SR-CRSP), condujeron una serie de estudios que generaron una valiosa base de datos que fue empleada en diversos trabajos de investigación, incluyendo el presente estudio. El archivo base, generado a partir de esta base de datos, comprendió inicialmente 5837 registros, que luego de su depuración se redujo a 3302. La información captada incluye datos de genealogía, de pesos vivos de corderos al nacimiento (PN), al destete a 5 meses (PD) y a la primera esquila a 8 meses (PE), así como la ganancia diaria de peso vivo del nacimiento al destete (GPND).

El análisis preliminar de los datos, permitió definir en los modelos la inclusión de los componentes de los efectos fijos, año de nacimiento y sexo, y como covariables el peso de la madre al empadre (para PN y PD), la edad del cordero al destete (para PD) y la edad a la primera esquila (para PE). Los componentes de covarianza fueron estimados mediante ASReml (Gilmour et al., 2006), en análisis univariado y bivariado.

Los modelos usados para la estimación de los parámetros genéticos en análisis univariado fueron:

$\mathrm{y}=\mathrm{Xb}+\mathrm{Z}_{1} \mathrm{a}+\mathrm{e}$;

$\mathrm{y}=\mathrm{Xb}+\mathrm{Z}_{1} \mathrm{a}+\mathrm{Z}_{2} \mathrm{~m}+\mathrm{e}, \operatorname{con} \operatorname{Cov}(\mathrm{a}, \mathrm{m})=0$;

$\mathrm{y}=\mathrm{Xb}+\mathrm{Z}_{1} \mathrm{a}+\mathrm{Z}_{2} \mathrm{~m}+\mathrm{e}, \operatorname{con} \operatorname{Cov}(\mathrm{a}, \mathrm{m})=\mathrm{Aó}_{\mathrm{am}} ;(\mathrm{M} 3)$

$\mathrm{y}=\mathrm{Xb}+\mathrm{Z}_{1} \mathrm{a}+\mathrm{e}+\mathrm{Z}_{\mathrm{z}} \mathrm{m}+\mathrm{Z}_{3} \mathrm{c}+\mathrm{e}$; 
Cuadro 1. Estimados de heredabilidad para los pesos vivos al nacimiento (PN), destete (PD), primera esquila (PE) y ganancia de peso al destete (GPND) de ovinos Junín, derivados del análisis univariado bajo el Modelo 2

\begin{tabular}{lcccc}
\hline & PN & PD & PE & GPND \\
\hline Heredabilidad directa $\left(\mathrm{h}^{2}\right)$ & $0.15 \pm 0.04$ & $0.14 \pm 0.04$ & $0.10 \pm 0.04$ & $0.15 \pm 0.04$ \\
Heredabilidad materna $\left(\mathrm{h}^{2}{ }_{\mathrm{m}}\right)$ & $0.24 \pm 0.04$ & $0.28 \pm 0.05$ & $0.13 \pm 0.06$ & $0.29 \pm 0.05$ \\
Heredabilidad total $\left(\mathrm{h}^{2}\right)$ & 0.27 & 0.28 & 0.16 & 0.29 \\
Log de la verosimilitud $(\log \mathrm{L})$ & -208.22 & -4190.89 & -4159.63 & 8652.92 \\
\hline
\end{tabular}

donde $\mathbf{y}$ es el vector de observaciones; $\mathbf{b}, \mathbf{a}$, $\mathbf{m}, \mathbf{c}$ y e son vectores de efectos fijos (b), y efectos aleatorios genético aditivo directo $(\mathbf{a})$, genético aditivo materno (m), de ambiente permanente materno $(\mathbf{c})$ y residual $(\mathbf{e})$; y $\mathbf{X}$, $\mathbf{Z}_{1}, \mathbf{Z}_{2}$ y $\mathbf{Z}_{3}$ son matrices de coeficientes que relacionan los efectos correspondientes de los vectores con $\mathbf{y}$. A es la matriz del numerador de parentesco entre animales, y $\mathbf{A} \sigma_{\mathrm{am}}$ es la covarianza entre efectos genético aditivo directo y maternal.

Los estimados de heredabilidad directa $\left(h_{a}^{2}\right)$, heredabilidad materna $\left(h_{m}^{2}\right)$ y el efecto de ambiente permanente materno $\left(c^{2}\right)$ fueron calculados como ratios de los estimados de $\sigma_{a}^{2}, \sigma_{m}^{2}$ y $\sigma_{c}^{2}$, respectivamente, en relación con la varianza total. La heredabilidad total $\left(h_{T}^{2}\right)$ fue estimada mediante el ratio entre $\left(\sigma_{a}^{2}+0.5 \sigma_{m}^{2}+1.5 \sigma_{\mathrm{am}}\right)$ y la varianza total (Willham, 1972). Para determinar la significancia de los efectos aleatorios en análisis univariado, se usó la prueba del ratioverosimilitud (LR) (Rao, 1973; Van Wyk et al., 2003; Ekiz, 2005).

Los modelos para el análisis bivariado fueron extensiones del univariado. Los estimados de correlación fenotípica $\left(r_{p_{1} p_{2}}\right)$, genética $\left(r_{a_{1} a_{2}}\right)$, maternal $\left(r_{m_{1} m_{2}}\right)$ y entre efectos genético directo y maternal $\left(r_{a_{1} m_{2}}\right)$

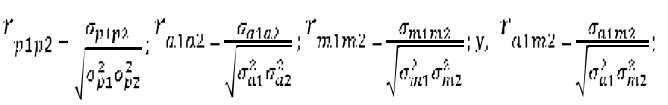

se obtuvieron a partir de las ecuaciones planteadas por Näsholm y Danell (1996) y Gilmour et al. (2006):

donde $\sigma_{p 1 p 2}, \sigma_{a 1 a 2}$, y $\sigma_{a 1 m 2}$ son las covarianzas fenotípica, genética aditiva directa, genética aditiva maternal, y entre los efectos genético directo y maternal, respectivamente; $\sigma_{p 1}^{2}$ у $\sigma_{p 2}^{2}, \sigma_{a 1}^{2}$ у $\sigma_{a 2}^{2}, \sigma_{m 1}^{2}$ y $\sigma_{m 2}^{2}$ son varianzas fenotípica, genética aditiva directa y maternal de las características 1 y 2 .

\section{Resultados}

\section{Análisis Univariado}

La prueba de LR indicó que el Modelo 2 fue el más adecuado para estimar los parámetros genéticos en el análisis univariado. Los valores de heredabilidad, derivados del análisis, se muestran en el Cuadro 1.

\section{Análisis Bivariado}

La prueba de LR indicó que el Modelo 3 fue el más adecuado para estimar los parámetros genéticos en el análisis bivariado. 
Cuadro 2. Estimados de heredabilidades y correlaciones entre peso al nacimiento (PN) con peso al destete (PD) y ganancia diaria de peso del nacimiento al de stete (GPND) y entre PD con GPND en ovinos Junín, derivados del análisis bivariado con el Modelo 3

\begin{tabular}{lccc}
\hline \multirow{2}{*}{ Parámetro $^{1}$} & \multicolumn{2}{c}{ PN con: } & PD con: \\
\cline { 2 - 4 } & PD & GPND & GPND \\
\hline $\mathrm{h}^{2}{ }_{\mathrm{a} 1}$ & $0.14 \pm 0.04$ & $0.16 \pm 0.04$ & $0.18 \pm 0.05$ \\
$\mathrm{~h}^{2}{ }_{\mathrm{a} 2}$ & $0.13 \pm 0.04$ & $0.15 \pm 0.04$ & $0.15 \pm 0.04$ \\
$\mathrm{~h}_{\mathrm{m} 1}^{2}$ & $0.25 \pm 0.08$ & $0.26 \pm 0.08$ & $0.23 \pm 0.09$ \\
$\mathrm{~h}_{\mathrm{m} 2}^{2}$ & $0.27 \pm 0.09$ & $0.23 \pm 0.09$ & $0.22 \pm 0.09$ \\
$\mathrm{r}_{\mathrm{p} 1 \mathrm{p} 2}$ & $0.35 \pm 0.02$ & $0.15 \pm 0.02$ & $0.98 \pm 0.00$ \\
$\mathrm{r}_{\mathrm{a} 1 \mathrm{a} 2}$ & $0.61 \pm 0.16$ & $0.48 \pm 0.17$ & $0.99 \pm 0.00$ \\
$\mathrm{r}_{\mathrm{m} 1 \mathrm{~m} 2}$ & $0.52 \pm 0.19$ & $0.31 \pm 0.23$ & $0.98 \pm 0.01$ \\
$\mathrm{r}_{\mathrm{a} 1 \mathrm{~m} 2}$ & $-0.34 \pm 0.34$ & $-0.39 \pm 0.35$ & $0.17 \pm 0.41$ \\
$\mathrm{r}_{\mathrm{a} 2 \mathrm{~m} 1}$ & $0.29 \pm 0.39$ & $0.28 \pm 0.34$ & $0.26 \pm 0.42$ \\
$\mathrm{r}_{\mathrm{a} 1 \mathrm{~m} 1}$ & $0.18 \pm 0.37$ & $-0.11 \pm 0.30$ & $0.16 \pm 0.39$ \\
$\mathrm{r}_{\mathrm{a} 2 \mathrm{~m} 2}$ & $0.11 \pm 0.40$ & $0.24 \pm 0.42$ & $0.27 \pm 0.44$ \\
Log L & -4175.53 & 7796.95 & 7470.53 \\
\hline
\end{tabular}

${ }^{1} \mathrm{~h}_{\mathrm{ai}}^{2}=$ heredabilidad directa de la i-ésima característica; $\mathrm{h}_{\mathrm{mi}}^{2}=$ heredabilidad materna de la i-ésima característica; $r_{\mathrm{plp} 2}=$ correlación fenotípica; $r_{\mathrm{a} 1 \mathrm{a2}}=$ correlación entre efectos aditivos directos; $r_{\mathrm{m} 1 \mathrm{~m} 2}=$ correlación entre efectos aditivos maternos; $r_{\text {aimj }}=$ correlación entre efecto genético aditivo directo de la i-ésima característica y efecto genético aditivo materno de la j-ésima característica; Log $L=$ logaritmo de verosimilitud. El Modelo 3 incluye los efectos genético aditivo directo, genético aditivo materno y la $\operatorname{cov}(a, m)=A \sigma_{a m}$

El Cuadro 2 presenta los estimados de heredabilidades y correlaciones derivados del análisis realizado, entre PN con PD y GP, y entre PD con GPND. Las demás combinaciones entre pares de características no se presentan porque presentaron valores indeterminados.

\section{Discusión}

\section{Análisis Univariado}

El estimado de $h_{a}^{2}$ para PN es inferior al valor de 0.31 obtenido mediante mínimos cuadrados reportado por Huapaya (1985), pero comparable con el estimado de 0.17 encontrado en ovinos de la misma raza por Bradford et al. (1989).

En los modelos que incluyen el efecto maternal (2, 3 y 4), los estimados de $h_{a}^{2}$ son comparables a los valores de 0.19 hallados en ovinos de doble propósito (Fogarty, 1995; Safari et al., 2005) y de 0.13 y 0.17 en las razas Romney Marsh y Corriedale, respectivamente (Martínez et al., 2006). Asimismo, el estimado de $h_{m}^{2}$ para PN es similar a los reportados por varios autores en ovinos de las razas Columbia, Merino, Targhee, Rambouillet y de doble propósito (Hanford $e t$ al., 2002, 2003; Cloete et al., 2003; Van Vleck 
et al., 2003; Snowder et al., 2004; Safari et al., 2005).

El estimado de $h_{a}^{2}$ para PD concuerda con el valor de 0.16 reportado previamente (Hanford et al., 2002; Safari et al., 2005; Martínez et al., 2006); asimismo, el estimado de $h_{m}^{2}$ para PD es superior a los valores de 0.08 a 0.20 hallados en las razas Corriedale, Romney Marsh y Hampshire (Hanford et al., 2002, 2003; Safari et al., 2005; Martínez et al., 2006). Sin embargo, los estimados de $h_{t}^{2}$ para PN y PD son comparables a los valores de 0.25 y 0.26 reportados por Martínez et al. (2006).

El estimado de $h_{a}^{2}$ para PE es similar al reportado por Huapaya (1985), pero inferior a los valores referidos por Bradford et al. (1989) y Safari et al. (2005). Igualmente, $h_{m}^{2}$ es similar a los estimados encontrados por Safari et al. (2005) y Ozder et al. (2009). Los estimados de $h_{a}^{2}$ y $h_{m}^{2}$ para GPND concuerdan con los reportados por Huapaya (1985) y Snowder y Van Vleck (2003), respectivamente.

\section{Análisis Bivariado}

En general, los estimados de $h_{a}^{2}$ y $h_{m}^{2}$ no sufrieron cambios respecto al análisis univariado. Sin embargo, la GPND influyó favorablemente en el estimado de $h_{a}^{2}$ la del PD, y la $h_{m}^{2}$ de la GP fue afectada desfavorablemente al haberse estimado en conjunto con PN y PD. Contrariamente, la $h_{a}^{2}$ de PN derivada del análisis con GPND fue más baja que las reportadas en análisis univariado por Bromley et al. (2000) en ovinos Columbia (0.25 vs 0.18$)$, Rambouillet (0.24 vs 0.19$)$ y Targhee ( 0.26 vs 0.22$)$. También, contrario a los resultados del presente estudio, reportan estimados de $h_{m}^{2}$ similares de los derivados de los análisis univariado y bivariado.

Los estimados de $\mathrm{r}_{\mathrm{a} 1 \mathrm{2} 2} \mathrm{y} \mathrm{r}_{\mathrm{m} 1 \mathrm{~m} 2}$ variaron entre valores de moderado a alto, y los correspondientes a $\mathrm{r}_{\mathrm{ml} \mathrm{m} 2}-$ entre PN con PD y GPND
- fueron negativos, indicando un posible antagonismo genético. Finalmente, los valores obtenidos para $\mathrm{r}_{\mathrm{ala} 2}, \mathrm{r}_{\mathrm{mlm} 2} \mathrm{y}_{\mathrm{a} 2 \mathrm{ml}}$, entre PN y PD, son comparables con lo reportado por Hanford et al. (2002), excepto para $\mathrm{r}_{\mathrm{a} 1 \mathrm{~m} 2}$.

\section{Conclusiones}

- El estimado de efecto genético aditivo materno fue importante y de magnitud moderada en la expresión de las características de crecimiento temprano, disminuyendo después del destete.

- El promedio de ganancia diaria de peso vivo del nacimiento al destete contribuyó a un mejor estimado de heredabilidad directa del peso al destete en análisis bivariado.

- Los estimados de índice de herencia de las características de crecimiento variaron de baja a moderada, pero las correlaciones genéticas y fenotípicas entre estas alcanzaron valores de moderados a altos, posibilitando su uso en programas de mejoramiento genético de la raza y la factibilidad de mejorar la velocidad de crecimiento mediante la selección indirecta de cualquiera de ellas.

\section{Literatura Citada}

1. Bahreini Behzadi MR, Amini A, Aslaminejad AA, Tahmoorespour M. 2013. Estimation of genetic parameters for production traits of Iranian Holstein dairy cattle. Livestock Research for Rural Development. Volume 25, Article \#156. [Internet]. Disponible en: http:// www.lrrd.org/lrrd25/9/bahr25156.htm

2. Bradford GE, Burfening PJ, Cartwright TC. 1989. Evaluation of production and reproduction of sheep, goat and alpaca genotypes in the Small Ruminant Collaborative Research Support Program. J Anim Sci 67: 30583067. 
3. Bromley CM, Snowder GD, Van Vleck LD. 2000. Genetic parameters among weight, prolificacy, and wool traits of Columbia, Polypay, Rambouillet, and Targhee sheep. J Anim Sci 78: 846-858.

4. Cloete SWP, Olivier JJ, Van Wyk JB, Erasmus GJ, Schoeman SJ. 2003. Genetic parameters and trends for birth weight, birth coat score and weaning weight in Merino lines divergently selected for ewe multiple rearing ability. S Afr J Anim Sci 33: 248-256. doi: 10.4314/sajas.v33i4.3781

5. Ekiz B. 2005. Estimates of maternal effects for pre- and post-weaning daily gain in Turkish Merino lambs. Turk J Vet Anim Sci 29: 399-407.

6. Fogarty NM. 1995. Genetic parameters for live weight, fat and muscle measurements, wool production and reproduction in sheep: a review. Anim Breed Abstr 63: 101-143.

7. Ghafouri-Kesbi F, Baneh H. 2012. Genetic parameters for direct and maternal effects on growth traits of sheep. Archiv Tierzucht 55: 603-611.

8. Gilmour AR, Gogel BJ, Cullis BR, Thompson R. 2006. ASReml User Guide Release 2.0. Hemel Hempstead, UK: VSN International Ltd.

9. Hanford KJ, Van Vleck LD, Snowder GD. 2002. Estimates of genetic parameters and genetic change for reproduction, weight, and wool characteristics of Columbia sheep. J Anim Sci 80: 3086-3098.

10. Hanford KJ, Van Vleck LD, Snowder GD. 2003. Estimates of genetic parameters and genetic change for reproduction, weight, and wool characteristics of Targhee sheep. J Anim Sci 81: 630-640.

11. Huapaya G. 1985. Parámetros genéticos provenientes de ovinos Corriedale y Junín por Corriedale. Tesis de Magister. Lima: Universidad Nacional Agraria La Molina. 119 p.

12. Linares $G$, Santiago B, Mellisho $E$. 2011. El ovino de la raza Junín. SAIS Túpac Amaru Limitada $N^{\circ} 1$. Registros
Genealógicos Zootécnicos del Perú. Lima, Perú: Universidad Nacional Agraria La Molina. $10 \mathrm{p}$.

13. Martínez A, Vásquez R, Vanegas J, Suárez M. 2006. Parámetros genéticos de crecimiento y producción de lana en ovinos usando la metodología de modelos mixtos. Rev Corpoica Cienc Tecnol Agropec 7(1): 42-49.

14. Momoh OM, Rotimi EA, Dim NI. 2013. Breed effect and non-genetic factors affecting growth performance of sheep in a semi-arid region of Nigeria. $\mathbf{J}$ Appl Biosciences 67: 5302-5307.

15. Mueller J. 2001. Mejoramiento genético de las majadas patagónicas. En: Ganadería sustentable en la Patagonia Austral. Borrelli P, Oliva G (eds). Ed INTA Reg Pat Sur. p 209-222.

16. Näsholm A, Danell O. 1996. Genetic relationships of lamb weight, maternal ability and mature ewe weight in Swedish finewool sheep. J Anim Sci 74: 329-339.

17. Ozder M, Sezenler T, Onal AR, Ceyhan A. 2009. Genetic and nongenetic parameter estimates for growth traits in Turkish Merino lambs. J Anim Vet Adv 8: 1729-1734.

18. Rao CR. 1973. Linear statistical inference and its applications. $2^{\text {nd }} e d$. New York, USA: John Wiley and Sons. $625 \mathrm{p}$.

19. Safari E, Fogarty NM, Gilmour AR. 2005. A review of genetic parameter estimates for wool, growth, meat and reproduction traits in sheep. Livest Prod Sci 92: 271-289. doi: 10.1016/ j.livprodsci.2004.09.003

20. Sánchez R. 1989. Las SAIS de Junín y la alternativa comunal. En: Debate Agrario: Análisis y Alternativas $\mathrm{N}^{\circ}$ 7. Lima: Centro Peruano de Estudios SocialesCEPES. p 85-101.

21. Snowder GD, Hanford KJ, Van Vleck LD. 2004. Comparison of models including cytoplasmic effects for traits of Rambouillet sheep. Livest Prod Sci 90: 159-66. doi: 10.1016/j.livprodsci. 2004.03.003 
22. Snowder GD, Van Vleck LD. 2003. Estimates of genetic parameters and selection strategies to improve the economic efficiency of postweaning growth in lambs. JAnim Sci 81:2704-2713.

23. Valdivia C, Pichihua J. 1986. El proceso de acumulación de capital, desarrollo y contradicciones internas de las SAIS en la sierra central. En: Perú: El problema agrario en debate. Lima Perú: Memorias SEPIA I. p 153-180.

24. Van Vleck LD, Snowder GD, Hanford KJ. 2003. Models with cytoplasmic effects for birth, weaning, and fleece weights, and litter size at birth for a population of Targhee sheep. J Anim Sci 81: 61-67.

25. Van Wyk JB, Fair MD, Cloete SWP. 2003. Revised models and genetic parameter estimates for production and reproduction traits in the Elsenburg Dormer sheep stud. S Afr J Anim Sci 33: 213-222. doi: 10.4314/sajas. v33i4.3777

25. Willham RL. 1972. The role of maternal effects in animal breeding: III. Biometrical aspects of maternal effects in animals. J Anim Sci 35: 128. 